\title{
Extension to the Messinger Model for Aircraft Icing
}

\author{
Tim G. Myers* \\ Cranfield University, Cranfield, England MK43 OAL, United Kingdom
}

\begin{abstract}
A one-dimensional mathematical model is developed describing ice growth due to supercooled fluid impacting on a solid substrate. When rime ice forms, the ice thickness is determined by a simple mass balance. The leadingorder temperature profile through the ice is then obtained as a function of time, the ambient conditions, and the ice thickness. When glaze ice forms, the energy equation and mass balance are combined to provide a single first-order nonlinear differential equation for the ice thickness, which is solved numerically. Once the ice thickness is obtained, the water height and the temperatures in the layers may be calculated. The method for extending the one-dimensional model to two and three dimensions is described. Ice growth rates and freezing fractions predicted by the current method are compared with the Messinger model. The Messinger model is shown to be a limiting case of the present method.
\end{abstract}

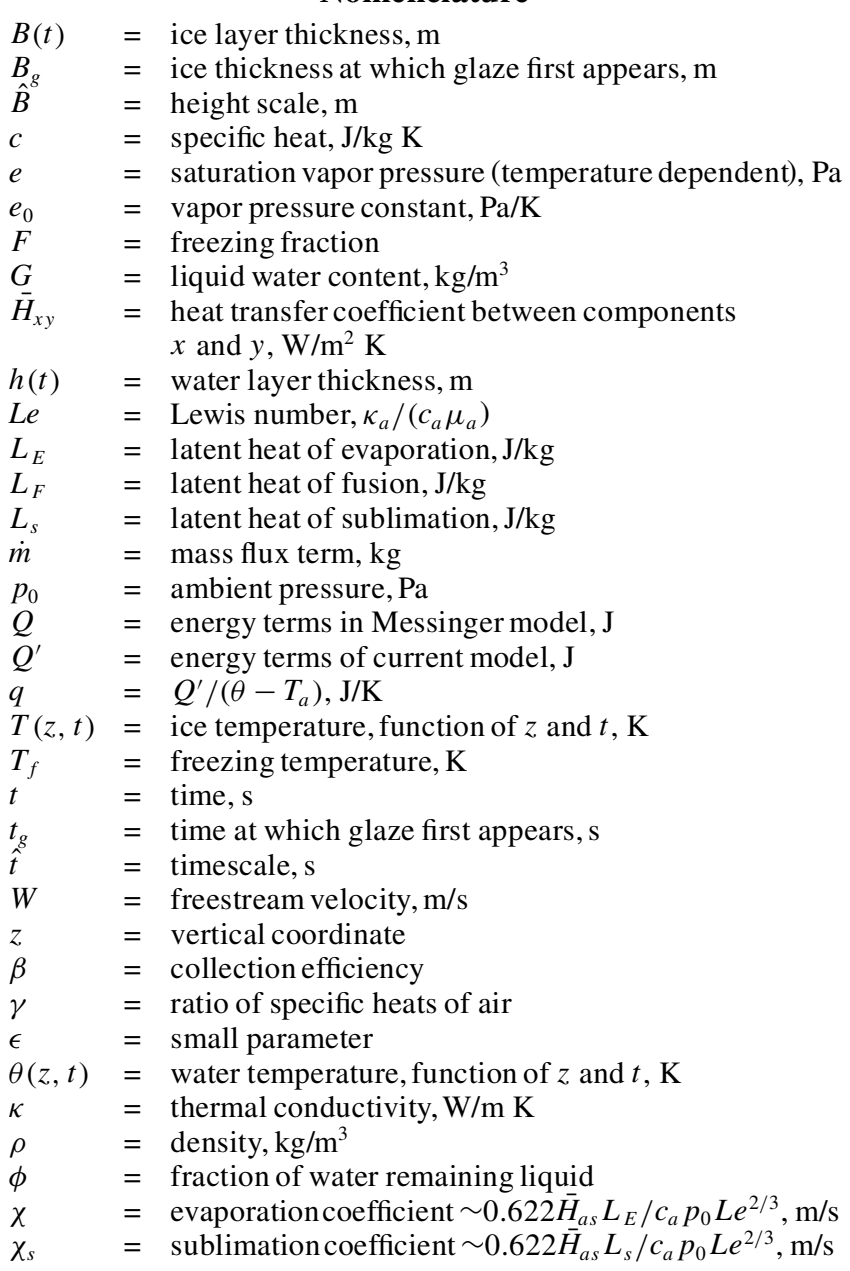

Subscripts

$\begin{array}{ll}a & =\text { air } \\ d & =\text { droplets } \\ g & =\text { glaze } \\ i & =\text { ice } \\ r & =\text { rime }\end{array}$

Received 14 April 1999; revision received 10 November 1999; accepted for publication 7 August 2000. Copyright (C) 2000 by Tim G. Myers. Published by the American Institute of Aeronautics and Astronautics, Inc., with permission.

${ }^{*}$ Senior Research Fellow, Applied Mathematics and Computing Group; t.g.myers@cranfield.ac.uk.

$\begin{array}{ll}s & =\text { substrate } \\ w & =\text { water }\end{array}$

\section{Introduction}

T $\mathrm{CE}$ growth on aircraft has long been recognized as a fundamental problem in the aerospace industry. Consequently, numerous methods have been developed to combat the problem, such as the use of freezing point depressants, thermal melting, and surface deformation. ${ }^{1}$ All of these have various drawbacks including the need to compromise on design to install such a system or simply high running costs. For example, a typical thermal antiicing system for along-range passenger jet consumes of the order of $10^{5} \mathrm{~W}$ when active.

As part of the ongoing battle to combat and understand the problem of ice accretion, a number of ice prediction codes have beendeveloped, such as LEWICE, TRAJICE, ONERA, and subsequent improvements. ${ }^{2-5}$ These codes are extremely complex, in that airflow, droplet trajectories, and a variety of parameters must be computed before commencing the icing calculation. Essential to this icing calculation is the energy balance, first set out by Messinger. Subsequent enhancements to the Messinger model, such as the inclusion of temperature rise due to compressible flow or the addition of an energy source at the substrate, have improved the model's applicability, ${ }^{2,7,8}$ However, the basic form of the model has not altered since its inception. The purpose of the present work is to improve on this model.

The Messinger ${ }^{6}$ model in its original form is a one-dimensional, equilibrium energy balance, designed to analyze "the conditions that govern the equilibrium temperature of an insulated, unheated surface exposed to icing." Because the temperature is set to its equilibrium value, the transient behavior of an ice accretion cannot not be captured. For example, in a one-step icing calculation, at the transition from rime ice to glaze ice growth the freezing fraction, defined by Messinger, switches instantaneouslyfrom the rime value of unity to another constant value, less than unity, and retains this new value for all time. In reality the freezing fraction will decrease monotonically from its initial rime value of unity to its final, equilibrium value. This is well known from studies of ice accretion based on the Stefan approach (for example, see Refs. 9 and 10). The freezing fraction predicted by Messinger ${ }^{6}$ is, therefore, always less than the true freezing fraction. Another limitation of the Messinger model is that the ice and water layers are isothermal, and so conduction through these layers cannot be accounted for. The substrate is assumed insulated, and so conduction is removed there also. With glaze ice, due to the flow and impact of incoming droplets, the water layer may be well mixed, in which case the isothermal assumption may be approximately valid; this will not be the case in the ice layer. Because this model does not allow heat to be conducted away from the ice/water interface, the energy in this region can only be balanced by the latent heat production as ice forms. In reality, the 
supercooled water and the subfreezing ice layer will permit conduction and so cool the interface, leading to an increase in ice production. For these reasons, the Messinger model must always predict as lower rate of ice accretion than occurs in practice. In certain cases of interest, this discrepancy may be small, but, as the current work will show, conduction is in general important in the ice accretion process.

The importance of conduction through the ice has been recognized by a number of workers (for example, see Refs. 10-12). In Ref. 11, the approach adopted by Messinger ${ }^{6}$ is employed, namely, to add an energy term to a static balance. This improves on the Messinger approximation but does not truly reflect the conduction. The approach adopted in Refs. 10 and 12 and related works correctly incorporates conduction and the effect of a phase change. In Ref. 12, a finite difference scheme is employed to solve a set of onedimensional partial differential equations describing the heat flow through a composite layer including phase change. In Ref. 10, the authors employ a finite element method, developed in Ref. 13, to solve the two-dimensional system of equations. The scheme runs on a supercomputer. Because of the model's complexity it is unlikely to be a simple addition to an icing code.

The work described in the current paper forms part of the ICECREMO project, to develop a three-dimensional aircraft icing code (see Acknowledgment). The method developed involves solving heat equations in the ice and water layers. At the moving ice/water interface, the energy balance leads to a phase change or Stefan condition, ${ }^{9,14}$ which takes the form of a first-order ordinary differential equation. This contains all of the terms of the standard Messinger ${ }^{6}$ model, as well as conductionterms. In general it requires numerical solving. The initial equations describing the thermal problem are equivalent to those described in Refs. 10 and 13. However, in the following work it is shown that it is not necessary to solve the full, complex system of equations. A much simpler system, which exploits that the ice growth rate is considerably slower than the heat conduction rate, adequately describes the problem. This method is sufficiently simple to allow it to be used in icing codes in much the same way the Messinger ${ }^{6}$ model is currently used. Furthermore, the method may be easily adapted to three dimensions, including a model for runback water. This is currently carried out in the prototype ICECREMO code. A simple method for adapting the model to deal with flowing water is described in Sec. VII. A much more computationally expensive method, which is unlikely to be suitable for an icing code, is described in Ref. 15.

In the following section the Messinger ${ }^{6}$ model is described in detail. In Sec. III, the mathematical formulation of the problem is provided. The energy terms of the Messinger model may then be seen as providing boundary conditions for the mathematical problem. In Sec. IV, the equations appropriate to rime ice growth are solved approximately. The glaze ice problem is dealt with in Sec. V. Formulas are given for the ice thickness and time when glaze first appears In Sec. VI, the solution method is detailed, and results showing the growth of ice and water over time are presented. These results are shown to compare well with experimental data. The method by which the one-dimensionalmodel may be extended to deal with two and three dimensions is described in Sec. VII. The glaze model is compared to the Messinger model in Sec. VIII. This shows that the two approaches are similar, with the exceptions that the Messinger model neglects conduction terms and cannotbe used to find temperature profiles. The importance of conduction is demonstratedand the Messinger model is shown to be a limiting case of the current model.

\section{Messinger ${ }^{6}$ Model}

The basis of the Messinger ${ }^{6}$ model is an energy balance. This balance relies on equating the heat lost from the ice and water accretion to the air and the production of latent heat due to ice growth. The mechanisms for losing energy are 1) convective heat transfer at the water surface, $\left.Q_{c}=\bar{H}_{a w}\left(T_{w}-T_{a}\right) ; 2\right)$ evaporative heat loss, $Q_{e}=\chi\left[e\left(T_{w}\right)-e\left(T_{a}\right)\right]$; and 3$)$ cooling by incoming droplets, $Q_{d}=\beta W G c_{w}\left(T_{w}-T_{d}\right)$. Those for gaining energy are 1) kinetic energy of incoming droplets, $\left.Q_{k}=(\beta W G) W^{2} / 2 ; 2\right)$ release of latentheat, $Q_{l}=\rho L_{F}(\partial B / \partial t)$; and 3$)$ aerodynamicheating, $Q_{a}=r \bar{H}_{a w} W^{2} / 2 c_{a}$.
The air temperature in $Q_{c}$ and $Q_{e}$ is frequently replaced by the dry adiabatic recovery. The recovery temperature is a consequence of the air compressibility; it is described in detail in Refs. 2, 4, 7, and 8 . However, in the following work, the notation is considerably reduced by the use of a single temperature in the air, droplets, and boundary layer. This restriction is easily removed. Water flow will also affect the heat balance; this is discussed in Sec. VII. Further information on energy sources in a freezing system may be found in Refs. 1, 16, and 17.

Balancing all of the energy terms leads to

$$
Q_{c}+Q_{e}+Q_{d}=Q_{k}+Q_{l}+Q_{a}
$$

From this heat balance, an estimate for the freezing fraction $F$ (the mass fraction of water that will turn to ice as it encounters the aircraft surface) can be obtained by setting the temperature in the ice and water layers to zero. A freezing fraction between 0 and 1 is taken as correct and used in subsequent calculations for glaze ice growth, with the average temperature held at zero degrees. If the calculation returns a freezing fraction greater than 1 , then only rime ice will occur. Because the freezing fraction is physically restricted to values between 0 and 1 , it is reset to 1 and the average temperature recalculated. Similarly, if the freezing fraction is found to be less than zero, then it is taken as identically zero and the (above freezing) average temperature estimated.

In aircraft icing codes, the icing calculation represents only one part of the problem. It is also necessary to calculate the airflow and droplet trajectories; the method is detailed in Refs. 1, 2, and 8. Hence all of the given energy terms are known, having being determined as part of the computational fluid dynamics (CFD) calculation. In the present work, these values will be assumed to be constant. In reality as the ice grows the values may vary and so may require recalculating and the new values employed in the energy balance. Taking constant parameter values could be viewed as dealing with only a single step of a multi-time-step calculation, and the values would subsequently be adjusted to new constants for the following time step.

\section{Mathematical Model}

Although the Messinger ${ }^{6}$ model has been used for a number of years to predict aircraft ice growth, a more rigorous mathematical formulation should provide significantly more information. For example, solving the heat equations in the ice and water will give detailed information on the temperature profiles and so improve the heat transfer estimates. An exact formula for the ice thickness at which the transition from rime to glaze occurs may also be obtained.

\section{A. Model Assumptions}

To simplify the problem, a number of assumptions will now be made

1) The evaporation function $e(T)$, required for $Q_{e}$, is approximated by a sixth-order polynomial in Ref. 18 over the range $T_{a} \in$ $[233,320] \mathrm{K}$. Over the range $[257,273.15]$ a good linear approximation to this curve is

$$
e(T) \approx-6.803 \times 10^{3}+e_{0} T
$$

where $e_{0}=27.03$. This is accurate to within $8 \%$ over the specified range.

2) The physical properties of the ice and water do not vary with temperature. However, the ice density will be allowed to take two distinct values, $\rho_{r}$ and $\rho_{g}$, depending on whether rime or glaze ice forms. The transition between the two is assumed to occur instantaneously.

3) The substrate temperature is fixed (and known). This requires the substrate to have high conductivity and a thermal mass much greater than that of the ice formation.

4) The droplet temperature, air temperature, and recovery temperature are taken as equal. This restriction may be easily altered.

5 ) The phase change occurs at a single temperature $T_{f}$.

These assumptions leave the aerodynamic heating and kinetic energy terms unchanged from the preceding section. The convective 
heat transfer, evaporation, and cooling by incoming droplets terms may now be expressed in the form

$$
\begin{gathered}
Q_{c}^{\prime}=\bar{H}_{a w}\left[\theta(B+h, t)-T_{a}\right]=q_{c}\left[\theta(B+h, t)-T_{a}\right] \\
Q_{e}^{\prime}=\chi e_{0}\left[\theta(B+h, t)-T_{a}\right]=q_{e}\left[\theta(B+h, t)-T_{a}\right] \\
Q_{d}^{\prime}=\beta W G c_{w}\left[\theta(B+h, t)-T_{a}\right]=q_{d}\left[\theta(B+h, t)-T_{a}\right]
\end{gathered}
$$

Primes are employed to distinguish the current energy terms, which involve the as yet unknown air/water interface temperature, from those of the Messinger ${ }^{6}$ model, which assumes a constant water temperature, $\theta=T_{w}$.

For airflow over ice, the convective heat transfer and evaporation terms become

$$
\begin{array}{r}
Q_{c i}^{\prime}=\bar{H}_{a i}\left[T(B, t)-T_{a}\right]=q_{c i}\left[T(B, t)-T_{a}\right] \\
Q_{s}^{\prime}=\chi_{s} e_{0}\left[T(B, t)-T_{a}\right]=q_{s}\left[T(B, t)-T_{a}\right]
\end{array}
$$

where $\chi_{s}$ is the sublimation coefficient. The aerodynamic heating term also changes,

$$
Q_{a i}=r \bar{H}_{a i} W^{2} / 2 c_{a}
$$

\section{B. Problem Formulation}

The following analysis is based on the standard method of specifying a phase change or Stefan problem. Stefan problems are used extensively in the investigation of melting or solidification. Mathematically equivalent problems arise in the study of diffusion and chemical reactions. 9,14

Consider the situation shown in Fig. 1. An ice layer of thickness $B(t)$ rests on top of a solid substrate. For the case of glaze ice, a water layer of thickness $h(t)$ will cover the ice. The temperature in the ice and water is denoted by $T(z, t)$ and $\theta(z, t)$, respectively. In the following analysis the model for glaze ice growth will be specified because most of the rime ice analysis may be recovered by setting the water thickness $h$ to zero in the appropriate equations. Whenever any other difference appears, the correct form for rime ice will also be given.

The Stefan problem is governed by four equations: heat equations in the ice and water, a mass balance, and a phase change or Stefan condition at the ice/water interface:

$$
\begin{gathered}
\frac{\partial T}{\partial t}=\frac{\kappa_{i}}{\rho_{i} c_{i}} \frac{\partial^{2} T}{\partial z^{2}} \\
\frac{\partial \theta}{\partial t}=\frac{\kappa_{w}}{\rho_{w} c_{w}} \frac{\partial^{2} \theta}{\partial z^{2}} \\
\rho_{i} \frac{\partial B}{\partial t}+\rho_{w} \frac{\partial h}{\partial t}=\beta W G \\
\rho_{i} L_{F} \frac{\partial B}{\partial t}=\kappa_{i} \frac{\partial T}{\partial z}-\kappa_{w} \frac{\partial \theta}{\partial z}
\end{gathered}
$$

Note that the ice density $\rho_{i}$ in Eqs. (4) and (5) may take different values depending on whether rime or glaze ice forms. In the current work, only two distinct values, $\rho_{r}$ and $\rho_{g}$, will be employed, and the transition between the two is assumed to be instantaneous.More complex formulas may be employed; however, care should be taken when integrating Eqs. (4) and (5) if these values are time dependent.

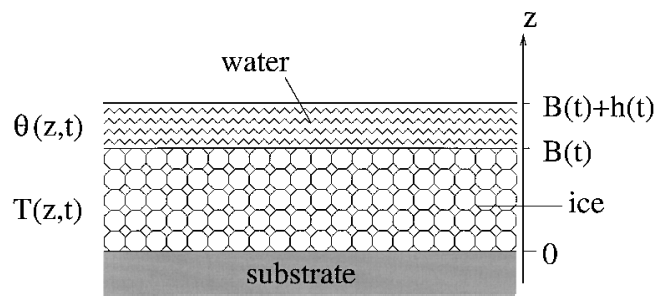

Fig. 1 Schematic of the ice and water system.
The Stefan condition (5) is a straightforward energy balance. It may also be interpreted as stating that the velocity of the phase change boundary is proportional to the temperature gradients across the boundary. The Stefan condition is similar to the Messinger ${ }^{6}$ energy balance in the form of a differential equation. However, unlike the Messinger model, this approach requires knowledge of the temperature gradients in each layer. Hence the heat equations (2) and (3) must be analyzed to solve the problem.

To determine the temperature in each layer, boundary and initial conditions must first be specified. These are based on the following assumptions:

1) The ice is in perfect thermal contact with the substrate, which has high conductivity and a thermal mass much greater than that of the ice accretion:

$$
T(0, t)=T_{s}
$$

2) The temperature is continuous at the phase change boundary and equal to the freezing temperature:

$$
T(B, t)=\theta(B, t)=T_{f}
$$

This condition is not imposed for the case of rime ice. For generality, the freezing temperature $T_{f}$ will be left unspecified throughout the following analysis; however, in all numerical calculations its value is set to $273.15 \mathrm{~K}$.

3) At the air/water interface, a standard radiation boundary condition with an added heat flux is imposed, ${ }^{19}$ stating that the heat flux at the surface is determined by convection, heat from incoming droplets, evaporation, aerodynamic heating, and kinetic energy:

$$
\begin{gathered}
-\kappa_{w} \frac{\partial \theta}{\partial z}=\left(Q_{c}^{\prime}+Q_{e}^{\prime}+Q_{d}^{\prime}\right)-\left(Q_{a}+Q_{k}\right) \\
-\kappa_{w} \frac{\partial \theta}{\partial z}=\left(q_{c}+q_{e}+q_{d}\right)\left(\theta-T_{a}\right)-\left(Q_{a}+Q_{k}\right), \quad z=B+h
\end{gathered}
$$

For rime ice the equivalent boundary condition is

$$
\begin{gathered}
-\kappa_{i} \frac{\partial T}{\partial z}=\left(Q_{c i}^{\prime}+Q_{s}^{\prime}+Q_{d}^{\prime}\right)-\left(Q_{a}+Q_{k}\right) \\
-\kappa_{i} \frac{\partial T}{\partial z}=\left(q_{c i}+q_{s}+q_{d}\right)\left(T-T_{a}\right)-\left(Q_{a i}+Q_{k}+\beta W G L_{F}\right) \\
z=B
\end{gathered}
$$

4) The substrate is initially clean:

$$
B=h=0, \quad t=0
$$

These conditions are sufficient to determine fully the temperature profiles and ice and water layer thicknesses.

Note that all of the terms of the Messinger ${ }^{6}$ energy balance have now appeared, either in the Stefan energy balance or in the boundary conditions.

\section{Rime Ice Growth}

The ice thickness in a rime calculation follows trivially from the mass balance, Eq. (4), with $h$ set to zero:

$$
B=\left(\beta W G / \rho_{r}\right) t
$$

The temperature profile may be obtained by considering an asymptotic expansion of Eq. (2). Taking only the leading order term will give a sufficiently accurate solution provided the lower-order correction terms are small.

To determine the asymptotic solution, denote a typical height scale $\hat{B}$; the corresponding timescale $\hat{t}$ is determined from Eq. (13). Using these scales to nondimensionalizeEq. (2) leads to

$$
\frac{\partial^{2} \bar{T}}{\partial \bar{z}^{2}}=\frac{\beta W G c_{i} \hat{B}}{\kappa_{i}} \frac{\partial \bar{T}}{\partial \bar{t}}=\epsilon \frac{\partial \bar{T}}{\partial \bar{t}}
$$

where overbars denote dimensionless quantities. 
Table 1 Parameter values used for Figs. 2-5

\begin{tabular}{lc}
\multicolumn{2}{c}{ for Figs. 2-5 } \\
\hline \hline Parameter & Value \\
\hline$c_{a}, \mathrm{~J} / \mathrm{kg} \mathrm{K}$ & 1014 \\
$c_{i}, \mathrm{~J} / \mathrm{kg} \mathrm{K}$ & 2050 \\
$c_{w}, \mathrm{~J} / \mathrm{kg} \mathrm{K}$ & 4218 \\
$e_{0}, \mathrm{~Pa} / \mathrm{K}$ & 27.03 \\
$G, \mathrm{~kg} / \mathrm{m}^{3}$ & 0.001 \\
$\bar{H}_{a w}, \mathrm{~W} / \mathrm{m}^{2} \mathrm{~K}$ & 500 \\
$\bar{H}_{i s}, \mathrm{~W} / \mathrm{m}^{2} \mathrm{~K}$ & 1000 \\
$\kappa_{i}, \mathrm{~W} / \mathrm{m} \mathrm{K}$ & 2.18 \\
$\kappa_{w}, \mathrm{~W} / \mathrm{m} \mathrm{K}$ & 0.571 \\
$L e$ & 13.4 \\
$L_{F}, \mathrm{~J} / \mathrm{kg}$ & $3.344 \times 10^{5}$ \\
$L_{E}, \mathrm{~J} / \mathrm{kg}$ & $2.26 \times 10^{6}$ \\
$p_{0}, \mathrm{~Pa}$ & $6.3 \times 10^{4}$ \\
$W, \mathrm{~m} / \mathrm{s}$ & 90 \\
$\beta$ & 0.55 \\
$\rho_{g}, \mathrm{~kg} / \mathrm{m}^{3}$ & 917 \\
$\rho_{r}, \mathrm{~kg} / \mathrm{m}^{3}$ & 880 \\
$\rho_{w}, \mathrm{~kg} / \mathrm{m}^{3}$ & 1000 \\
$\chi, \mathrm{m} / \mathrm{s}$ & 11.0 \\
\hline \hline
\end{tabular}

Equation (14) may be solved in the form of a series in powers of $\epsilon$, provided $\epsilon \ll 1$. This occurs if the typical height of the accretion satisfies

$$
\hat{B} \ll \kappa_{i} / \beta W G c_{i}
$$

Taking typical values for the constants, given in Table 1, indicates $\kappa_{i} / \beta W G c_{i}=2.4 \mathrm{~cm}$, that is, the series solution will be valid for ice thicknesses considerably less than $2.4 \mathrm{~cm}$. The leading-order problem, in dimensional form, is then specified by

$$
\frac{\partial^{2} T}{\partial z^{2}}=0
$$

First- and higher-order corrections will not be investigated in the present work. Equation (16) indicates that the leading-order temperature profile is linear in $z$. It may, however, be nonlinear in time, which enters through the moving boundary conditions. Integrating Eq. (16) twice and applying conditions (6) and (11), gives

$$
T=T_{s}+\frac{Q_{a i}+Q_{k}+\beta W G L_{F}-\left(q_{c i}+q_{d}+q_{s}\right)\left(T_{s}-T_{a}\right)}{\kappa_{i}+B\left(q_{c i}+q_{d}+q_{s}\right)} z
$$

The reduction of the heat equation (2) from a partial differential equation to an ordinary differential equation [Eq. (16)] produces what is termed the quasi- or pseudosteady problem because time only enters through the moving boundary conditions, applied at $z=B(t)$. The physicalinterpretation of this is that the timescale for ice growth, determined by the amount of incoming fluid, is much slower than that for conduction through the ice. Therefore the temperature has time to equilibrateas the ice slowly accumulates.Quasisteady problems typically arise when the region of interest is thin. Examples may be found in numerous physical situations including reaction-diffusion systems ${ }^{20}$ or viscous flow. ${ }^{21,22}$ They are a particular example of a singular perturbation (for example, see Ref. 23) because neglecting the time derivative means that the solution becomes invalid as $t \rightarrow 0$ and an initial condition may not be satisfied. This singular limit is not considered in the current analysis.

\section{Glaze Ice Growth}

With the substrate temperature fixed below freezing and supercooled droplets impacting on the surface, the ice growth must occur in two distinct stages. During the first stage, all of the incoming water freezes almost instantaneously, whereas in the second stage, both water and ice develop simultaneously. This was also noted in Ref. 10. The physical reason for this two-stage process is that the model requires good heat transfer between the substrate and incoming fluid. The initial incoming water must, therefore, immediately adopt the subfreezing substrate temperature and, because it has a nucleation site, freeze. Only when there is a sufficiently thick insulating layer and enough energy has been introduced into the system can water appear. This will be demonstrated mathematically later in this section. Note that this layer may be extremely thin in relatively mild conditions and involve only a fraction of an initial impacting droplet. The freezing of even a very small proportion of a droplet may provide sufficient latent heat for the remainder to heat up and stay liquid and flow over the surface as runback. This is a consequence of the assumption that the droplets are supercooled and the substrate has a fixed, subzero temperature.

Using a similar argument to that in the preceding section, the heat equations may be simplified to quasi-steady forms:

$$
\frac{\partial^{2} T}{\partial z^{2}} \approx 0, \quad \frac{\partial^{2} \theta}{\partial z^{2}} \approx 0
$$

provided

$$
\begin{gathered}
\hat{B} \ll \frac{\kappa_{i}}{(1-\phi) \beta W G c_{i}} \sim \frac{0.024}{1-\phi} \mathrm{m} \\
\hat{h} \ll \frac{\kappa_{w}}{\phi \beta W G c_{w}} \sim \frac{0.003}{\phi} \mathrm{m}
\end{gathered}
$$

where $\phi$ is the fraction of water that remains liquid, and so the argument leading to Eq. (16) is strengthened, with $2.4 \mathrm{~cm}$ being a conservative estimate for the maximum limit of the ice thickness. The water layer, however, must remain thin (unless there is a slow buildup of water, $\phi \ll 1$, which allows time for the temperature to adjust), with $3 \mathrm{~mm}$ being the lower value of the estimate. This is still well within the limit of water thicknesses expected in aircraft icing conditions.

If the water and ice layers do satisfy the preceding inequalities, the temperature in the ice is

$$
T=\left[\left(T_{f}-T_{s}\right) / B\right] z+T_{s}
$$

where Eqs. (6) and (7) have been imposed. The temperature in the water is

$$
\theta=T_{f}+\frac{Q_{a}+Q_{k}-\left(q_{c}+q_{d}+q_{e}\right)\left(T_{f}-T_{a}\right)}{\kappa_{w}+h\left(q_{c}+q_{d}+q_{e}\right)}(z-B)
$$

where Eqs. (7) and (9) have been imposed.

In the earlier rime ice example, the ice thickness was obtained from the mass balance without knowledge of the temperature profile. In the present case, the problem is coupled; the temperatures specified by Eqs. (19) and (20) depend on the ice and water heights, which in turn depend on the temperature through Eq. (5).

To solve the coupled problem, first integrate the mass balance (4) to obtain an expression for $h$ as a function of $B$ and $t$ :

$$
h=\left(\beta W G / \rho_{w}\right)\left(t-t_{g}\right)-\left(\rho_{g} / \rho_{w}\right)\left(B-B_{g}\right)
$$

This involves the constants of integration $B_{g}$ and $t_{g}$, the ice thickness and time at which water first appears. These are determined by a continuity argument, which is most easily followed after an expression has been obtained for the ice growth rate.

Differentiating Eqs. (19) and (20) provides expressions for the temperature gradients, which, together with Eq. (21) may be substituted into the Stefan condition, Eq. (5), to provide a first-order nonlinear ordinary differential equation for the ice thickness:

$$
\begin{array}{r}
\rho_{g} L_{F} \frac{\partial B}{\partial t}=\frac{\kappa_{i}\left(T_{f}-T_{s}\right)}{B}-\kappa_{w} \frac{Q_{a}+Q_{k}-\left(q_{c}+q_{d}+q_{e}\right)\left(T_{f}-T_{a}\right)}{\kappa_{w}+h\left(q_{c}+q_{d}+q_{e}\right)} \\
=\frac{\kappa_{i}\left(T_{f}-T_{s}\right)}{B} \\
-\kappa_{w} \rho_{w} \frac{Q_{a}+Q_{k}-\left(q_{c}+q_{d}+q_{e}\right)\left(T_{f}-T_{a}\right)}{\kappa_{w} \rho_{w}+\left[\beta W G\left(t-t_{g}\right)-\rho_{g}\left(B-B_{g}\right)\right]\left(q_{c}+q_{d}+q_{e}\right)}
\end{array}
$$

The rime ice model of the preceding section has the ice growth rate specified by Eq. (13); the water thickness and growth rate are 
both zero. For a smooth transition from the rime to glaze models, the ice and water thicknesses and growth rates must be continuous. To determine when this occurs, the ice mass growth rate from the mass balance (4) is substituted into the Stefan condition (5) to give

$$
\begin{aligned}
& L_{F}(\beta W G)=\kappa_{i}\left(T_{f}-T_{s}\right) / B_{g} \\
& \quad-\left[Q_{a}+Q_{k}-\left(q_{c}+q_{d}+q_{e}\right)\left(T_{f}-T_{a}\right)\right]
\end{aligned}
$$

Rearranging this provides an expression for $B_{g}$ :

$$
B_{g}=\frac{\kappa_{i}\left(T_{f}-T_{s}\right)}{\beta W G L_{F}+\left[Q_{a}+Q_{k}-\left(q_{c}+q_{d}+q_{e}\right)\left(T_{f}-T_{a}\right)\right]}
$$

This is the ice thickness at which glaze will first appear. This explicit formula shows how $B_{g}$ depends on the ambient conditions. An important feature of the equation is that it allows positive, negative, and even infinite values for $B_{g}$. These may be interpreted in the following way. If $0<B_{g}<\infty$, then Eq. (25) indicates the ice thickness at which glaze first appears. An infinite or negative value for $B_{g}$ indicates glaze ice will never appear. This could occur either because the numerator $T_{f}-T_{s}<0$, that is, the substrate is too warm for any ice to grow. Alternatively, the denominator of Eq. (25) is less than or equal to zero, in which case there will never be enough energy in the system to produce water, and the accretion is pure rime, for all time. This is discussed in greater detail in Sec. VIII.

Because the ice thickness must be continuous between the two growth regimes, the time when glaze first appears is determined by comparison with Eq. (13):

$$
t_{g}=\left(\rho_{r} / \beta W G\right) B_{g}
$$

An a priori proof that the assumption $T(0, t)=T_{s}$ requires initial rime ice growth is as follows. If, instead of initial rime ice growth, glaze or water appears first, then the initial conditions on the mass balance Eq. (4) and the Stefan condition (23) are $B=h=0$. Equation (23) shows $\partial B / \partial t \rightarrow \infty$, due to the $1 / B$ dependence. To conserve mass, Eq. (4) then indicates that $\partial h / \partial t \rightarrow-\infty$. Because $h$ is initially zero, this indicates that the water height immediately becomes negative. This is clearly unphysical, and the only reasonable assumption to draw is that when glaze ice first appears $B=B_{g}>0$. However, this restriction on $B_{g}$ may not be very strong; for example, in moderate conditions $B_{g}$ may be extremely small and the initial rime phase may only contribute a small fraction of the total ice accretion. An earlier work, which includes a model for heat transfer between the ice and substrate, permits solutions in which initially glaze ice or even pure water may develop. ${ }^{24}$

\section{Solution Method and Results}

The method for solving an ice accretion problem under known ambient conditions proceeds as follows

1) Calculate all of the necessary parameter values and determine the ice thickness and time at which glaze first appears, $B_{g}, t_{g}$, via Eqs. (25) and (26).

2) If $B_{g} \leq 0$, this indicates the model assumptions are invalid and glaze ice never forms. Alternatively, if $t_{g}$ is less than the required exposure time to icing conditions, $t_{\exp }$, there will not be sufficien time for glaze to form. In either case, the ice thicknessis determined by Eq. (13), the temperature throughout the ice is given by Eq. (17), and the calculation is terminated here

3) If $B_{g}>0$ and $t_{g}<t_{\text {exp }}$, then glaze ice will occur during the icing calculation. The ice thickness is determined by numerical integration of Eq. (23). Once $B(t)$ is known, the water thickness and the ice and water temperatures are given by Eqs. (21), (19), and (20), respectively.

\section{A. Ice Growth Results}

To demonstrate the described method, two icing calculations are shown in Fig. 2. These are for air temperatures of 270 and $263.15 \mathrm{~K}$. The substrate temperature in both examples is assumed to be the same as the air temperature. Two solid lines are shown in Fig. 2; these show the ice height and ice plus water height when $T_{a}=263.15 \mathrm{~K}$. For small times, the lines coincide because initially there is no water present, $h=0$. The lines diverge only when water appears. In this

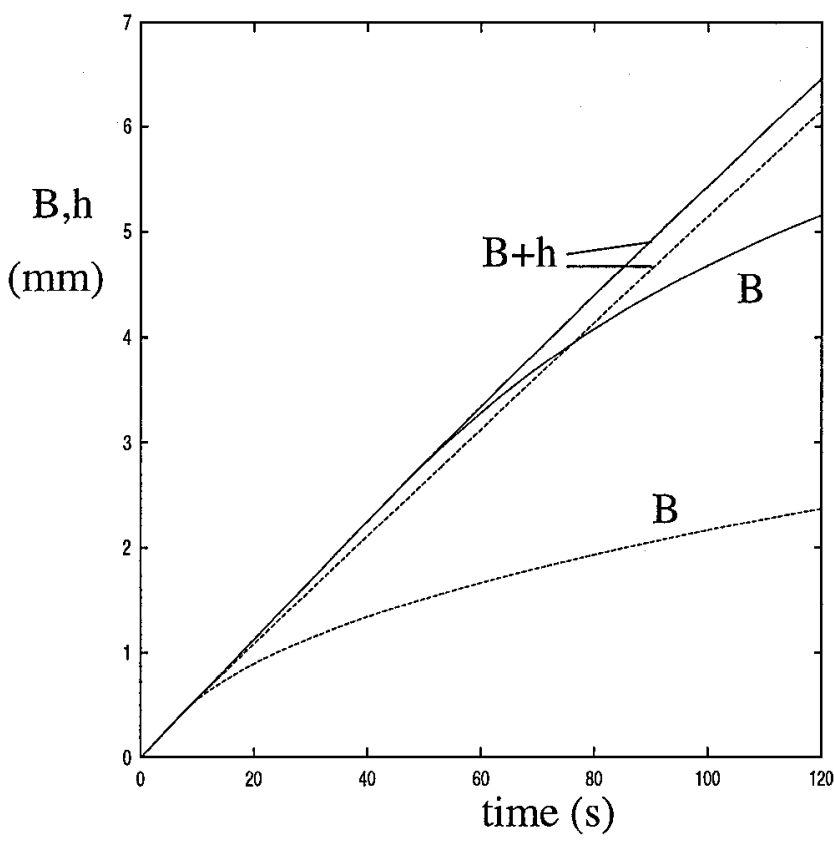

Fig. 2 Ice and water growth for $T_{a}=263.15 \mathrm{~K}(-)$ and $T_{a}=270 \mathrm{~K}$ $(-\ldots) ; T_{s}=T_{a}$ in both cases.

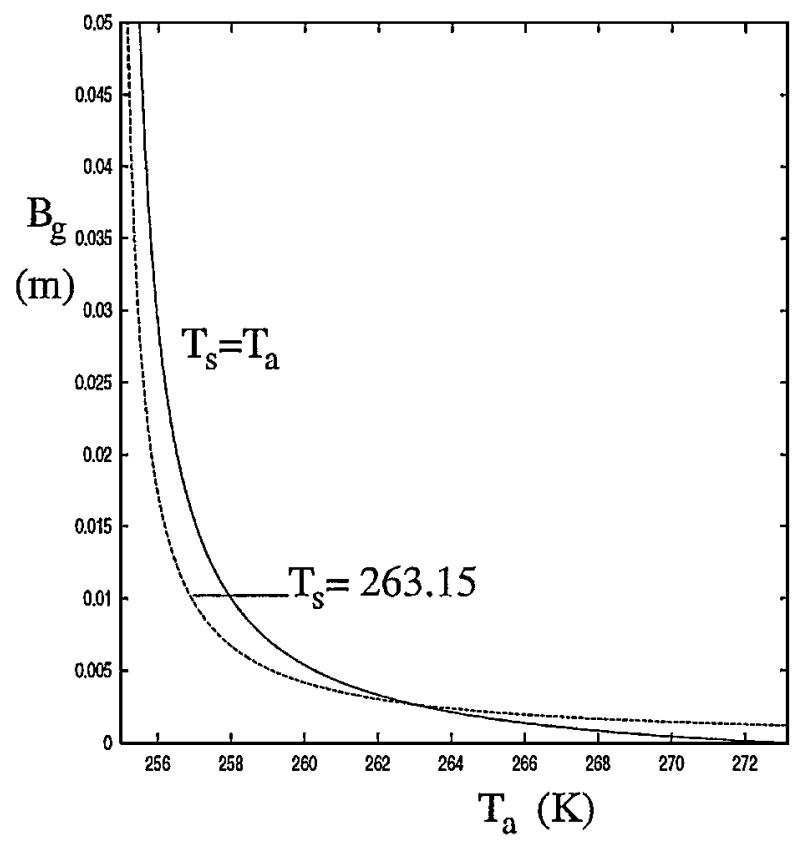

Fig. $3 B_{g}$ vs $T_{a}$ with $T_{s}=T_{a}\left(-(-)\right.$ and $T_{s}=263.15 \mathrm{~K}(-\ldots)$.

case, water and, hence, glaze ice first occur after $45.86 \mathrm{~s}$, when the ice height is $2.58 \mathrm{~mm}$. The broken lines show the same situation for an ambient temperature of $T_{a}=270 \mathrm{~K}$. Because the air is now much warmer, glaze appears much more rapidly, $t_{g}=7.95 \mathrm{~s}$, and the ice has a thickness of $B_{g}=0.45 \mathrm{~mm}$.

Figure 3 shows the variation of the time glaze first appears with ambient temperature. The solid line is for the case when the substrate temperature is the same as the air temperature. This shows $B_{g}=0$ when $T_{a}=T_{f}$. Because $B_{g}$ is the rime ice thickness at which glaze first appears and the model requires an initial ice layer, then when $T_{s}=T_{a}=T_{f}$, there can be no ice accretion. Decreasing $T_{a}$ increases the value of $B_{g}$ until a singularity is reached at $T_{a}=254.76$. This is the lowest temperature at which glaze ice can occur under the present conditions.

The broken curve in Fig. 3 shows the value of $B_{g}$ with a substrate temperature fixed below freezing, $T_{s}=263.15 \mathrm{~K}$. The value of the ambient temperature at which glaze never occurs is the same as for the preceding case. However, when $T_{a}=T_{f}$, the broken curve 


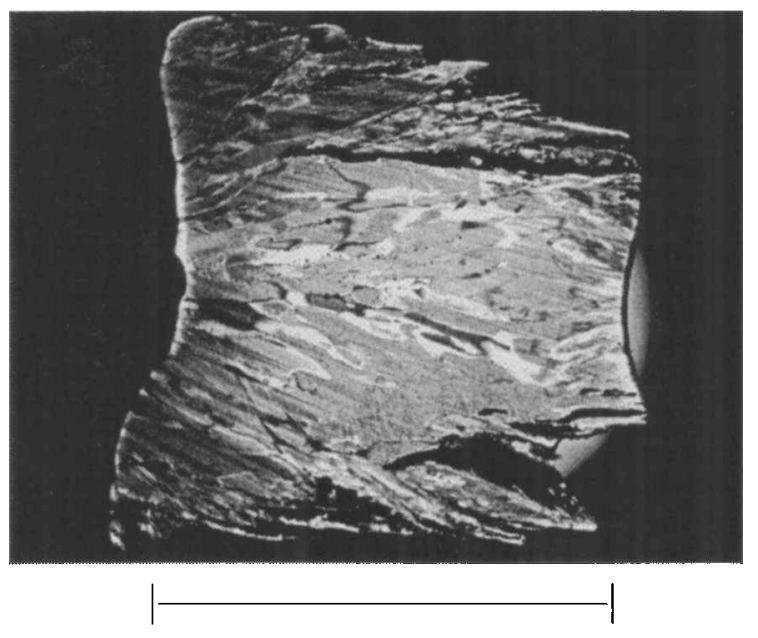

\section{$20 \mathrm{~mm}$}

Fig. 4 Microstructure of a piece of ice grown on a model aluminum airfoil (NACA 0012) at 263.15 K.

displays a nonzero $B_{g}$, which indicates ice may grow even when the air temperature is above freezing. This phenomenon could occur, for example, if the aircraft fuel remains cold after flying at a high altitude and it has not had sufficient time to heat up before the craft encounters water. The large thermal mass of the fuel may be sufficient to keep the wing below the ambient temperature for some time resulting in a surface temperature less than ambient. ${ }^{1}$

Qualitative experimental validation for the current model is given in Fig. 4. This shows a thin section of a piece of ice grown in the Aerospace Composite Technology Artington Icing Wind Tunnel (Guildford, England, United Kingdom) as part of the ICECREMO test program. The microstructureis highlighted by the use of a strong background light source, which may be partially seen to the right of the ice section. The accretion was formed in approximately $12 \mathrm{~min}$ on a NACA 0012 aluminum model, with dimensions $40 \times 4.8 \mathrm{~cm}$. The ice grew from right to left, into the airflow. Although some of the specimen has been broken away during the process of recoveringit, a layer of fine columnargrains, associated with rime ice growth, can be seen near the original growth front. As the accretion has continued to grow, the grain structure has become coarser, associated with glaze ice growth. The experimental conditions associated with Fig. 4 are specified in Table 1. A CFD calculation carried out using FLUENT version 5 , under the same conditions as those in the wind tunnel but in a freestream, that is, not confined in a tunnel, shows that the typical value for the catch efficiency at the leading edge is 0.55 , giving a mass flux $\beta W G=0.0495 \mathrm{~kg} \cdot \mathrm{m}^{2} \cdot \mathrm{s}$. As mentioned in the discussion of Fig. 2, Eq. (25) gives a value of $B_{g}=2.58 \mathrm{~mm}$. The height of the fine grain region in Fig. 4 varies between 2 and $3 \mathrm{~mm}$.

\section{Extension to Two and Three Dimensions}

The one-dimensional energy balance is specified by Eq. (23). To extend the model to two and three dimensions requires the addition of energy terms due to water flow into and out of the control volume. These terms will be denoted $Q_{\text {ent }}$ and $Q_{o}$. The convective energy terms $Q_{\text {ent }}$ and $Q_{o}$ act as an energy source and sink, respectively, in the conductive energy balance:

$$
\begin{aligned}
\rho_{g} L_{F} & \frac{\partial B}{\partial t}=\frac{\kappa_{i}\left(T_{f}-T_{s}\right)}{B}+\kappa_{w} \frac{\left(Q_{c}+Q_{e}+Q_{d}\right)-\left(Q_{a}+Q_{k}\right)}{\kappa_{w}+h\left(q_{c}+q_{d}+q_{e}\right)} \\
& +\left(Q_{o}-Q_{\mathrm{ent}}\right)
\end{aligned}
$$

This must be coupled with a mass balance,

$$
\dot{m}_{\mathrm{ent}}+\dot{m}_{\mathrm{inc}}=F\left(\dot{m}_{\mathrm{ent}}+\dot{m}_{\mathrm{inc}}\right)+\dot{m}_{e}+\dot{m}_{o}
$$

where $\dot{m}_{\text {ent }}$ and $\dot{m}_{o}$ are the water entering and leaving the control volume, respectively, $\dot{m}_{\text {inc }}$ is the mass influx due to incoming droplets, and $\dot{m}_{e}$ is the evaporation. For the standard Messinger ${ }^{6}$ energy balance, the combined mass and energy balance method is described in greater detail in Refs. 1-3 and references contained therein. In the presentcase, the icing calculation proceeds in exactly the same manner. Because the initial rime ice growth involvesno flow, Eq. (25) still determines the ice thickness at which water first appears. However, in this case the catch varies in space $\beta=\beta(\boldsymbol{x})$ and water first appears at the point where $B_{g}$ takes its minimum value. Finally, Eq. (27) may be simplified even further because the conduction term through the water $h\left(q_{c}+q_{d}+q_{e}\right)$ may be neglected for the following reason.

In the one-dimensional case, where there is no flow, a typical water layer thickness may be of the order of millimeters (as shown in Fig. 2). In this case, $h\left(q_{c}+q_{d}+q_{e}\right)$ is of a similar magnitude to $\kappa_{w}$. In a typical aircrafticing situation, the water will flow and is unlikely to reach such heights; heights of order $\frac{1}{10} \mathrm{~mm}$ are more realistic. ${ }^{15} \mathrm{In}$ this case, $h\left(q_{c}+q_{d}+q_{e}\right) \ll \kappa_{w}$, and conduction through the water may be neglected. This is equivalent to assuming infinite conduction through the water, and so the water remains at an approximately constant temperature. Because $\theta=T_{f}$ at $z=B$, then $\theta \approx T_{f}$ everywhere. This is the temperature used to determine the energy terms $Q_{\text {ent }}$ and $Q_{o}$. Further, as described in Sec. VIII, this is the temperature that is normally used in the Messinger ${ }^{6}$ model. The problem of ice growth in the presence of a flowing liquid is then governed by the mass balance (28) and the energy balance

$$
\begin{gathered}
\rho_{g} L_{F} \frac{\partial B}{\partial t}=\frac{\kappa_{i}\left(T_{f}-T_{s}\right)}{B}+\left(Q_{c}+Q_{e}+Q_{d}+Q_{o}\right) \\
-\left(Q_{a}+Q_{k}+Q_{\mathrm{ent}}\right)
\end{gathered}
$$

\section{Comparison with the Messinger ${ }^{6}$ Model}

In the following subsections the model developed in this paper is compared with the analytical result of Messinger. ${ }^{6}$ It is shown that for thin fluid films the large time limit of the current model is exactly the Messinger model. At intermediate times the Messinger model underpredicts the freezing rate.

\section{A. One-Dimensional Model}

During glaze ice formation, the Messinger ${ }^{6}$ model requires the water temperature to equal the freezing temperature $T_{f}$. Applying the linear approximation for $e(T)$, the convective, evaporative, and droplet cooling energy terms are

$$
\begin{gathered}
Q_{c}=\bar{H}_{a w}\left(T_{f}-T_{a}\right), \quad Q_{e}=\chi e_{0}\left(T_{f}-T_{a}\right) \\
Q_{d}=\beta W G c_{w}\left(T_{f}-T_{a}\right)
\end{gathered}
$$

The Messinger ${ }^{6}$ energy balance is then

$$
\rho_{g} L_{F} \frac{\partial B}{\partial t}=Q_{c}+Q_{e}+Q_{d}-\left(Q_{a}+Q_{k}\right)
$$

The current model has an ice growth rate specified by Eq. (22). With the definition of energy terms given by Eq. (30), this may be written

$$
\rho_{g} L_{F} \frac{\partial B}{\partial t}=\frac{\kappa_{i}\left(T_{f}-T_{s}\right)}{B}+\kappa_{w} \frac{\left(Q_{c}+Q_{e}+Q_{d}\right)-\left(Q_{a}+Q_{k}\right)}{\kappa_{w}+h\left(q_{c}+q_{d}+q_{e}\right)}
$$

A comparison of Eq. (31) with Eq. (32) highlights the differences between the two models.

First, the current model contains an extra term proportional to $1 / B$. This represents heat conduction through the ice. For small ice thicknesses, such as at the onset of accretion or in regions of low catch efficiency, this is likely to be the dominant term in the energy balance and the Messinger ${ }^{6}$ model will be inappropriate.

Second, the term on the right-hand side of Eq. (31) has a denominator of 1 , whereas in Eq. (32) the denominator is $1+$ $h\left(q_{c}+q_{d}+q_{e}\right) / \kappa_{w}$. The difference in terms represents conduction through the water layer. Typically, $\left(q_{c}+q_{d}+q_{e}\right) / \kappa_{w} \sim 2 \times 10^{3}$. For very thin films (for example, when the water is allowed to flow), $2 \times 10^{3} h \ll 1$, and conduction through the water layer will be so rapid that the water film may be neglected. The appropriate terms in Eqs. (31) and (32) are then approximately the same. In one dimension, the water film will typically be $\mathcal{O}\left(10^{-3}\right)$, and the water 
provides an insulating layer. For example, if cooling effects dominate, so that $Q_{c}+Q_{e}+Q_{d}>Q_{a}+Q_{k}$, then the ice growth rate specified by Eq. (32) decreases from that predicted by Eq. (31) as the water layer thickness increases. That is, the water layer insulates the ice accretion from the cool ambient conditions. If heating effects dominate, $Q_{c}+Q_{e}+Q_{d}<Q_{a}+Q_{k}$, the ice growth rate will increase from that predicted by Eq. (31) with increasing $h$.

The Messinger ${ }^{6}$ model assumes the water to be at a constant temperature, which is set to the freezing temperature $T_{f}$ for glaze ice calculations. The present analysis shows that this is the correct choice because the water layer affects the energy balance through the phase change boundary, where the temperature is $T_{f}$.

Equation (31) may be used to determine the conditions under which water will never appear. If no water appears, the ice mass growth rate is $\rho_{r} \partial B / \partial t=\beta W G$. The energy associated with this phase change must balance the remaining energy terms. Substituting this growth rate into Eq. (31) leads to an expression for the air temperature below which glaze never occurs:

$$
T_{a}=T_{f}-\frac{\beta W G L_{F}+Q_{a}+Q_{k}}{q_{c}+q_{d}+q_{e}}
$$

The current model may also be used to determine the conditions at which glaze never appears. Equation (25) gives the ice thickness at which the rime/glaze transition occurs. If this thickness tends to infinity, then water never appears. The ambient temperature at which this occurs is determined by setting the denominator of Eq. (25) to zero. Rearranging this expression then gives Eq. (33). The current model, therefore, agrees exactly with the Messinger ${ }^{6}$ model on the conditions at which glaze will not occur.

\section{B. Higher-Dimensional Model}

As discussed in Sec. VII, when the water is allowed to flow, the term that reflects the insulating effect of the water layer is negligible. In this case, the energy balance is given by Eq. (29). The Messinger model is given by Eq. (31), with the addition of $Q_{0}-Q_{\text {ent }}$ on the right-hand side. This must be coupled to the mass balance, Eq. (28). Now the only difference between the energy balances is the term representing conduction through the ice. The magnitude of this term depends on the ambient conditions and the ice thickness. This is highlighted in the following section. Note that when flow is allowed the water temperature is approximately the same as the freezing temperature $T_{f}$. The ice temperature is still determined by Eq. (19).

\section{Freezing Fractions}

The freezing fraction, defined by Messinger ${ }^{6}$ as the mass of ice divided by the mass of incoming fluid, has long been recognized as an important quantity in icing calculations:

$$
\begin{aligned}
F= & \frac{\rho_{r}}{\beta W G t} B, & t<t_{g} \\
& \frac{\rho_{r} B_{g}+\rho_{g}\left(B-B_{g}\right)}{\beta W G t}, & t>t_{g}
\end{aligned}
$$

Figure 5 shows three curves, representing the freezing fractions determined by the Messinger model (labeled curve a), by the current one-dimensional model (labeled $b$ ) and by the current method with $h \equiv 0$, as discussed in Sec. VII (labeled c). The ambient conditions are the same as those employed in Fig. 2 with $T_{a}=263.15 \mathrm{~K}$. However, the calculation is carried on for a longer time than in Fig. 2 to show clearly that curve $\mathrm{b}$ eventually falls below curve $\mathrm{a}$. The Messinger model has a constant ice growth rate, given by Eq. (31); therefore, the variation of $B$ is linear in time. Consequently, the freezing fraction is constant once water has appeared. Before this time, the ice growth rate is a different constant specified by the mass balance [Eq. (13)]. The current method has an ice growth rate dependent on the ice thickness and so decreases in time. Unlike the Messinger model, the switch in ice growth rates at $t=t_{g}$ is continuous and decreases monotonically. Close to $t=t_{g}$, it may be observed that the Messinger model ice growth rate is less than half the true value. Only after $3 \mathrm{~min}$ are curves a and $\mathrm{b}$ reasonably close. Note that the value predicted by the Messinger model is not the asymptote of

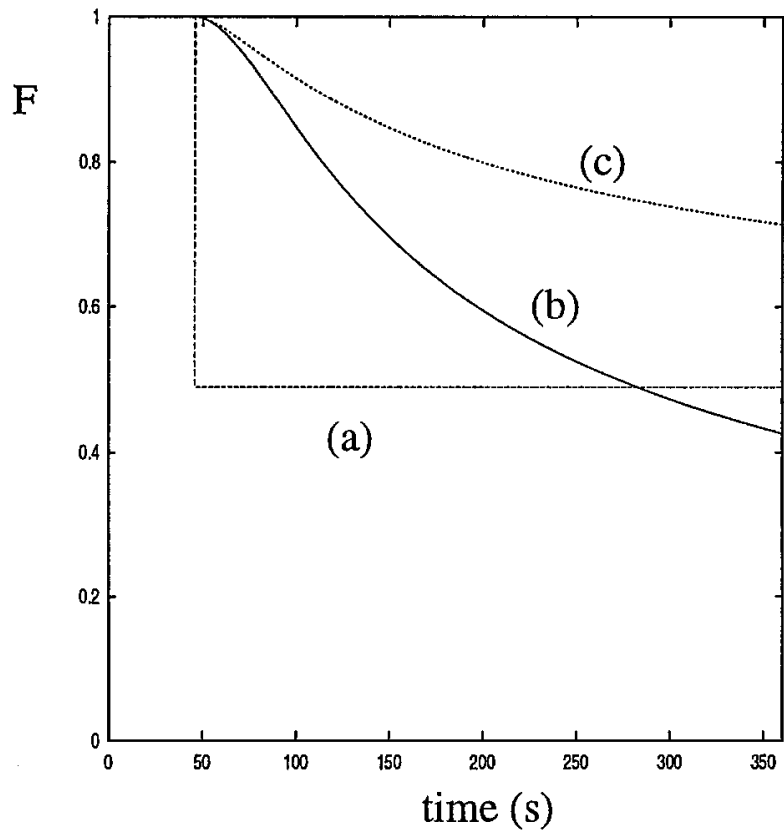

Fig. 5 Variation of freezing fraction with time for $T_{a}=T_{s}=263.15 \mathrm{~K}$; curve a, the Messinger model; curve b, current one-dimensional model; and curve c, current model with $h=0$.

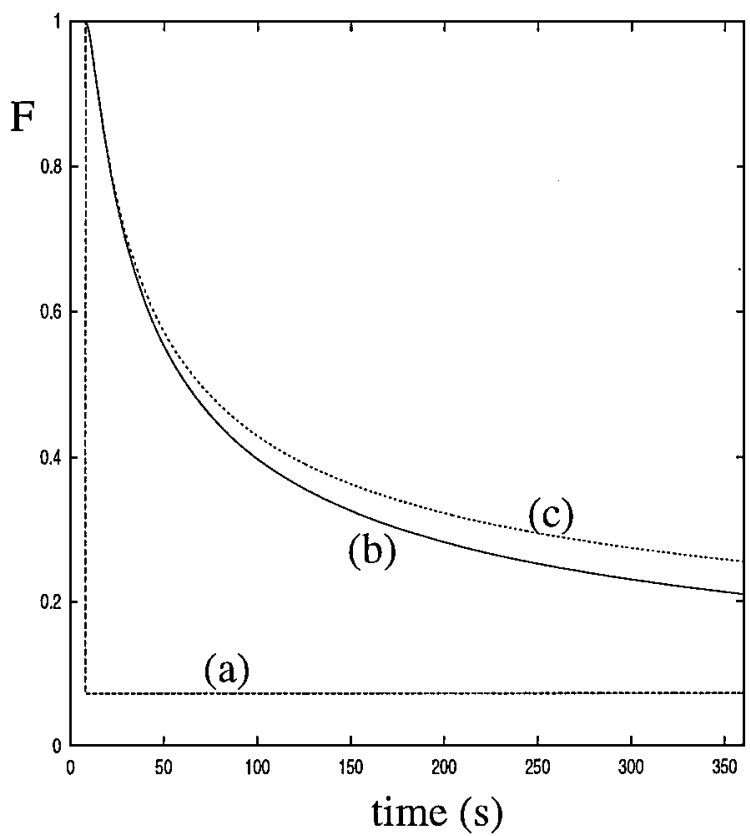

Fig. 6 Variation of freezing fraction with time for $T_{a}=T_{s}=270 \mathrm{~K}$; curve a, the Messinger model; curve b, current one-dimensional model; and curve c, current model with $h=0$.

curve b, which decreases below curve a after $280 \mathrm{~s}$. Curve c denotes the energy balance when $h \equiv 0$. This is the approximate solution when the fluid is allowed to flow. Curve c predicts a greater ice growth rate than curve a for all time. In fact, the Messinger model is the asymptote for curve c; however, it approaches this curve very slowly. After $360 \mathrm{~s}$ of ice accretion (in this case equivalent to $311 \mathrm{~s}$ of glaze ice growth) the ice growth rates still differ by $30 \%$.

Figure 6 shows the equivalent curves for an ambient temperature of $270 \mathrm{~K}$. Now the Messinger ${ }^{6}$ model underestimates the ice growth rate by 65 and $70 \%$ for the one-dimensional and water flow problems, respectively, after 360-s accretion time (352 s of glaze ice accretion)

Figures 5 and 6 demonstrate that the validity of the Messinger ${ }^{6}$ model decreasesas the temperature increases. The Messinger model is, therefore, most appropriate close to temperatures where water 
will not appear. For example, for the conditions specified in Table 1 , water will never appear below $T_{a}=254.8 \mathrm{~K}$. When $T_{a}=258 \mathrm{~K}$, the differences between the Messinger model freezing fraction and that obtained by the one-dimensional and flow models is 3.6 and $17 \%$ after $120 \mathrm{~s}$ of glaze ice accretion. Decreasing the temperature will bring the curves close together even more rapidly.

\section{Summary}

A mathematical model has been developed to determine ice and water growth in the presence of incoming supercooled droplets. For rime ice growth, a mass balance determines the ice thickness. Once this is obtained, the approximate temperature profile may be calculated. For glaze ice growth, the leading-orderproblem, for relatively thin layers, reduces to a single first-order differential equation for the ice thickness, which requires solving numerically. Once this has been achieved, quantities such as the water thickness and temperature profiles can be readily calculated.

With a fixed temperature at the substrate, the results show that rime ice will grow initially. This occurs because the substrate provides many nucleation points and so, because the initial water droplets must adopt the subzero substrate temperature, freezing takes place. Subsequently, provided conditions are sufficiently mild, a water layer will appear.

The main advantages of using the current approach are that it provides the temperature profiles in the ice and water and an exact formula for the ice thickness at which glaze is first observed Although it has not been proven experimentally, the current mode appears to give more physically sensible results than the Messinger model. For example, the Messinger model predicts an immediate switch from the constant rime ice freezing fraction $(F=1)$ to a second constant value $(F<1)$ when glaze appears. The current model predicts a smooth transition, with $F$ decreasing monotonically from 1. Because ice may conduct heat, it seems perfectly reasonable that the energy balance and, consequently, the freezing fraction should depend on the ice thickness. When the water layer is thin, $F$ tends toward the Messinger model value at large times. The difference between the two models is greatest when the ice layer is thin and conduction through the ice dominates.

The method employed assumes that the substrate has a known (fixed) temperature, and so conduction through the substrate is neglected. Physically, the current model is best applied to a substrate of high conductivity, such as metal, with a thermal mass that is large compared to that of the ice accretion. Because the method requires the numerical solution of a first-order differential equation, it is slightly more complicated than the Messinger ${ }^{6}$ model but still simple enough to be employed in full icing codes. The current model has already been adapted to three dimensions and coupled with a water flow model in the ICECREMO code.

The relative effect of the driving mechanisms behind the ice growth can be easily identified from this analysis. For pure rime ice growth, the ice height is determined by a simple mass balance. If ambient conditions are sufficiently mild the rime layer will turn to glaze. The time at which this transition takes place increases proportionally to the temperature difference between freezing and the substrate, $T_{f}-T_{s}$; with decreasing energy source terms, $Q_{a}+Q_{k}$, or increasing energy sink terms $Q_{c}+Q_{d}+Q_{e}$; and with decreasing catch or mass flux.

The subsequent glaze ice growth rate increases with increasing conduction through the ice, either due to decreasing substrate temperature or decreasing ice thickness; increasing water layer thickness if energy source terms dominate or decreasing water layerthickness if energy sink terms dominate; and increasing energy sink terms or decreasing energy source terms.

Perhaps one of the most important results of this analysis is the explicit formula for the ice thickness at which glaze first appears. Setting the denominator of this expression to zero will determine the mildest conditions at which glaze will never appear. The experimental evidence shown in Fig. 4 indicates that the formula gives physically realistic values.

By tackling this physical situation using a Stefan model, the present method can provide information on temperature distributions and freezing rates. The freezing rate, in particular, is a useful descriptorof local ice-forming conditions and has a direct bearing on the grain size, which may be measured directly. The grain structure in turn determines the ice strength. This type of model, therefore, provides significantly more information than does the energy balance method currently employed in icing codes.

\section{Acknowledgments}

The ICECREMO project (the development of three-dimensional ice accretion modeling) is a collaboration between British Aerospace, Rolls-Royce, GKN-Westland Helicopters, Ltd., and the Defence Evaluation and Research Agency. The project is managed by the British Aerospace and is part funded by the Department of Trade and Industry under Contract RA/6/31/05. I would like to thank D. W. Hammond of BAe Sowerby Research Centre for providing the photograph used in Fig. 4.

\section{References}

${ }^{1}$ Thomas, S. K., Cassoni, R. P., and MacArthur, C. D., "Aircraft AntiIcing and De-Icing Techniques and Modeling," Journal of Aircraft, Vol. 33, No. 5, 1996, pp. 841-854.

${ }^{2}$ Ruff, G. A., and Berkowitz, B. M., "Users Manual for the NASA Lewis Ice Accretion Prediction Code (LEWICE),” NASA CR 185129, May 1990.

${ }^{3}$ Potapczuk, M., "LEWICE/E: A Euler Based Ice Accretion Code," AIAA Paper 92-0037, 1992.

${ }^{4}$ Gent, R. W., "TRAJICE 2-A Combined Water Droplet Trajectory and Ice Accretion Prediction Program for Aerofoils," Royal Aircraft Establishment, RAE TR 90054, Farnborough, England, U.K., Nov. 1990.

${ }^{5}$ Hedde, T., and Guffond, D., "ONERA Three-Dimensional Icing Model,' AIAA Journal, Vol. 33, No. 6, 1995, pp. 1038-1045.

${ }^{6}$ Messinger, B. L., "Equilibrium Temperature of an Unheated Icing Surface as a Function of Air Speed," Journal of the Aeronautical Sciences, Jan. 1953, pp. 29-42

${ }^{7}$ Schlichting, H., Boundary-Layer Theory, 7th ed., McGraw-Hill, New York, 1979, Chap. 13

${ }^{8}$ Gent, R. W., and Cansdale, J. T., "Ice Accretion on Aerofoils in TwoDimensional Compressible Flow-A Theoretical Model," Royal Aircraft Establishment, RAE TR 82128, ICECREMO, Farnborough, England, U.K., Jan. 1983.

${ }^{9}$ Hill, J. M., One-Dimensional Stefan Problems: An Introduction, Longman Science Technical, Harlow, England, U.K., 1987, Chap. 1

${ }^{10}$ Huang, J. R., Keith, T. G., Jr., and De Witt, K. J., "Efficient Finite Element Method for Aircraft De-Icing Problems," Journal of Aircraft, Vol 30, No. 5, 1993, pp. 695-704

${ }^{11}$ Bourgault, Y., Beaugendre, H., and Habashi, W. G., "Development of a Shallow-Water Icing Model in FENSAP-ICE," Journal of Aircraft, Vol. 37 No. 4, 2000, pp. 640-646.

${ }^{12} \mathrm{De}$ Witt, K. J., and Baliga, G., "Numerical Simulation of OneDimensional Heat Transfer in Composite Bodies with Phase Change,"NASA CR-165607, 1982.

${ }^{13}$ Rolph, W. D., III, and Bathe, K.-J., "An Efficient Algorithm for Analysis of Nonlinear Heat Transfer with Phase Changes," International Journal for Numerical Methods in Engineering, Vol. 18, No. 1, 1982, pp. 119-134.

${ }^{14}$ Crank, J. D., Free and Moving Boundary Value Problems, Oxford Science, Oxford, 1984, Chap. 1.

${ }^{15}$ Myers, T. G., Charpin, J. P. F., and Thompson, C. P., "Slowly Accreting Glaze Ice Due to Supercooled Water Impacting on a Cold Surface," Physics of Fluids (submitted for publication)

${ }^{16}$ Poots, G. I., Ice and Snow Accretion on Structures, Research Studies, Taunton, England, U.K., 1996, pp. 74-77.

${ }^{17}$ Chilton, T. J., and Colburn, A. P., "Mass Transfer (Absorption) Coefficients," Industrial and Engineering Chemistry, Vol. 26, No. 11, 1934 pp. 1183-1187.

${ }^{18}$ Lowe, P. R., "An Approximating Polynomial for the Computation of Saturation Pressure," Journal of Applied Meteorology, Vol. 16, 1977, pp. 100103.

${ }^{19}$ Carslaw, H. S., and Jaeger, J. C., Conduction of Heat in Solids, Clarendon, Oxford, 1959, p. 19.

${ }^{20}$ Murray, J. D., Mathematical Biology, Springer-Verlag, Heidelberg, Germany, 1989, Chap. 5.

${ }^{21}$ Greenspan, H. P., "On the Motion of a Small Viscous Droplet That Wets a Surface," Journal of Fluid Mechanics, Vol. 84, No. 1, 1978, pp. 125-143.

${ }^{22}$ Moriarty, J. A., Schwartz, L. W., and Tuck, E. O., "Unsteady Spreading of Thin Liquid Films with Small Surface Tension," Physics of Fluids, Vol. A3, No. 5, 1991, pp. 733-742.

${ }^{23}$ Murray, J. D., Applied Mathematical Sciences Vol. 48: Asymptotic Analysis. Springer-Verlag, New York, 1984

${ }^{24}$ Myers, T. G., and Hammond, D. W., "Ice and Water Film Growth from Incoming Supercooled Droplets," International Journal of Heat and Mass Transfer, Vol. 42, 1999, pp. 2233-2242. 